\title{
تحضير فحم منشط من سعف النخيل كمادة مازة جديدة لإزالة حالات التسمم بعقار الميفيناميك أسيا
}

لقاء حسين علوان السامرائي كلية التربية / جامعة سامراء

الملخص

إنّ التسمم بفرط الجرعة الدوائية بعالج بمعلق الفحم المنشط الذي يمنز على سطحه الأدوية ويمنع امتصاصها ـ لذلك أجريت هذه الدراسة لتقدير قابلية الفحم المنشط المحضر من سعف النخيل كمادة مازة جديدة لعقار Mefenamic acid ( البونستان ).

استخدمت تقنية المطيافية فوق البنفسجية لمتابعة الكميات الممتزة بعد مزج محلول الدواء مع الفحم المنشط المحضر • ونت اجراء التجربة عند درجات حرارية مختلفة () C 45 , 35 , 25 , 15 , 10 وذلك لحساب الدوال الثرموداينمكية $\left(\Delta H^{\circ}, \Delta G^{o}, \Delta S^{\circ}\right.$

إن عملية امتزاز البونستان على سطح الفحم المنشط المحضر يكون عند التراكيز الواطئة وتزداد الكمية الممتزة بزيادة التراكيز الابتدائية للعقار وتتخفض كمية الامتزاز بزيادة درجة الحرارة ممّا بدلّ على أن التفاعل باعث للحرارة ـ وأوضحت الدراسة ان لتغيير الدالة الحامضية تأثنر على عملية الامثزاز .

\section{المقدمة :}

يعّد التسمم الدوائي Drug intoxication مشكلة كبيرة تواجهنا في عصرنا الحالي ، وذلك بسبب كثرة استخدام هذه الأدوية Pharmaceutical من قبل المجتمع وبطريقة سهلة للوصول إلى العديد منها سواء صنف واحد أو اكثر من هذه الأدوية ، ويعٌّ عقار Mefenamic acid احد الأدوية التي تسبب التسمم عند استخدامها بجرعات تقوق الجرعة الاعتيادية . 
فعند الثعور بالألم لأي شخص فانه مباشرة يتعاطى مسكنات خافضة للألم بدون اية وصفة من قبل

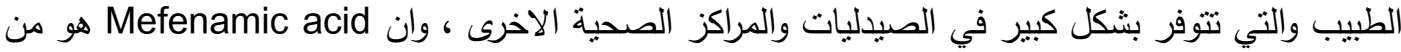
الأدوية المسكنة للألام Pain killers وبمفعول مركزي دون أن تؤثر على الوكي الوعي والاحساسات الأخرى

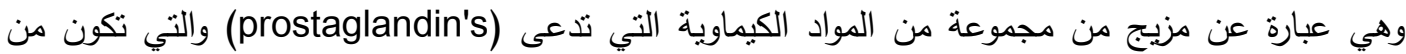

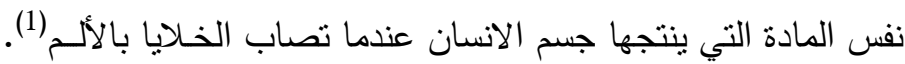

إنّ (Mefenamic acid) ويدعى أيضًا بالاسم التجاري (البونستان) وهو دواء خافض للحرارة ومسكن ومضاد للالتهاب ، يستخدم في معالجة بعض الالام كالصداع Headaches بانواعه وآلام الأسنان ، وهو

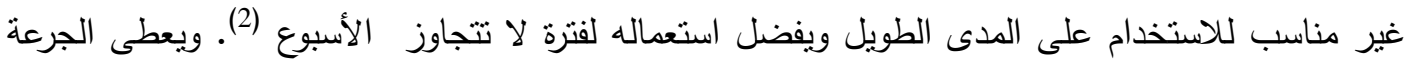
للبونستان عن طريق الفم وبمقدار 500mg ثلاثة مرات يوميا( كل ستة ساعات) .

والصيغة التركيبيه :

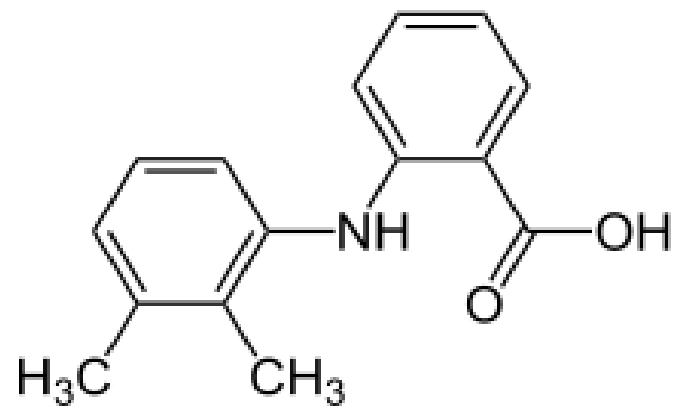

2-(2,3-dimethylphenyl)aminobenzoic acid

لقد أجريت دراسات بحثيه عديدة لأزاله حالات التسمم باستخدام سطوح مازه مختلفة لها القدرة و الكفاءة في معالجة حالات التسمم ببعض الأدوية منها الكاربون المنشط والكاؤلين ومخلفات الذرة الصفراء وغيرها .

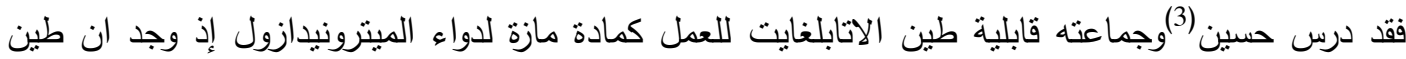
الاتابلغايت يسنطيع امتزاز دواء الميترونيدازول عند التزاكيز الواطئة جدا، ونقل كمية الامتزاز بزيادة درجة

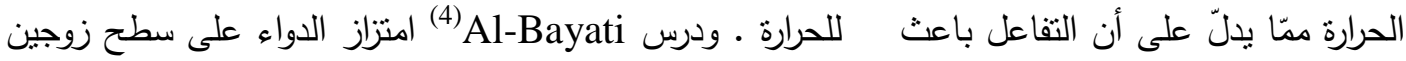
من المواد المازة وهي الاتابلغايت والسليلوز ووجد كفاءة عالية في سعة امتزاز العقار وتم تطبيق ايزوثثرم

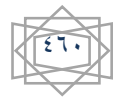


لانكماير وفرندلخ للاهتزاز • ودرس السامرائي (5) قابلية امتزاز الباراستول على سطح الكاؤلين إذ وجد له كفاءة امتزازه عالية عند التراكيز القليلة وتم حساب الدوال الثرموديناميكية للاهتزاز .

هدف هذه الدراسة هو إيجاد مادة مازة جديدة محضرة من مواد اولية متوافرة بكثرة لمعالجة حالات التسمم بالأدوية.

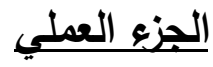

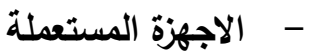

- - ميزان حساس باربع مراتب من نوع 2

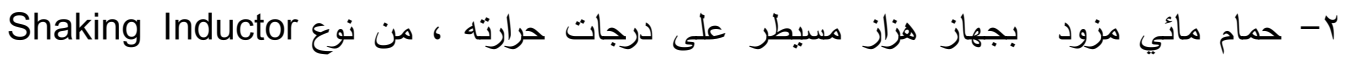
GCA./Precision Scientific Chicago,U.S.A

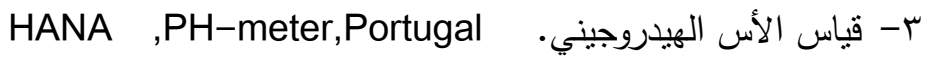

Shemadzu, U.V-100- وكان من نوع UV.-Visible قوعنية الأشعة المرئية- فوق البنفسية .02, U.V.-Visible ,Spectro- phtometer

\section{- - - - المواد وطرائق العمل :}

Mefenamic acid دواء البونستان

استعملت في هذه الدراسة دواء البونستان ، والذي جهز من الثركة العامة لصناعة الأدوية والمسنلزمات

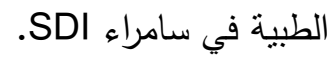

ץ- تحضير الفحم المنشط من سعف النخيل 
استخدم سعف نخلة التمر العراقية المتوفرة محليا كبديل لامتزاز حالات النسمم بالأدوية من محاليلها المائية حيث تم طحن الاجزاء اليابسة (السعف ) من مخلفات النظلة إلى قياس اقل أو يساوي ( 1 ملم ) ثم استخدم المسحوق لامتزاز المحاليل المحضرة مختبريا للعقار قيد الدراسة.

$$
\text { r- تحضير المحاليل وتحديد منحني المعايرة }
$$

حضر محلول قياسي من العقار المـراد دراسة أمتزازه على سطح الفحم المنشط المحضر من سعف

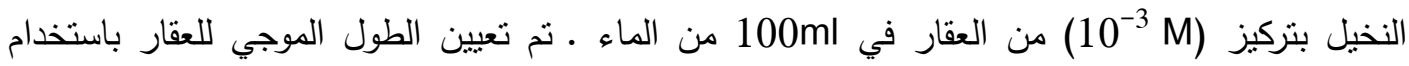
التركيز المناسب وبأستخدام جهاز تقنية الأشعة المرئية- فوق البنفسجية .UV. -Visb وذلك عند المدى

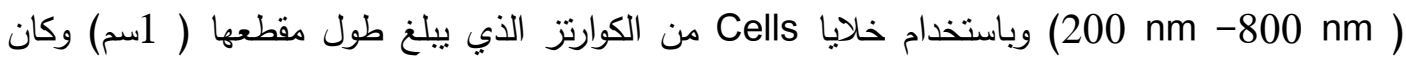
$\cdot(288 \mathrm{~nm})$

تم رسم منحني المعايرة بين الأمتصاصية Absorption والتركيز Concentration و والثنكل رقم (1) ، وهي يوضح هذه الدراسة ، وذلك من خلال تحضير نراكيز مختلفة

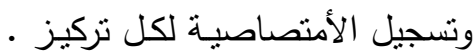

ع- ايجاد وتحديد الزمن اللازم للوصل إلى حالة الاتزان

من أجل إيجاد الزمن اللازم للوصول إلى حالة الاتزان ما بين السطح ألمـاز Adsorbent والمـادة

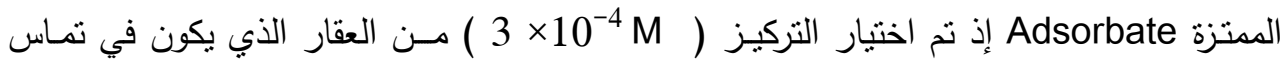

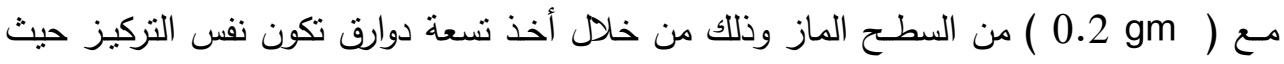

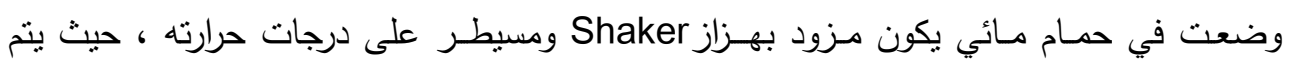

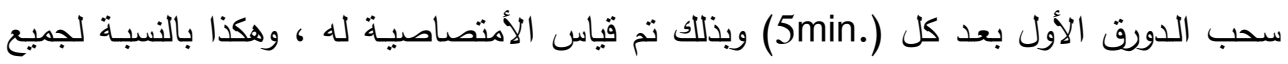

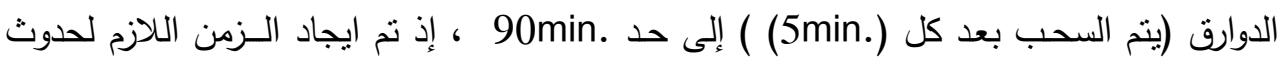
الاتزان وهو (70min ) ( النم ). 
درس أيزوثيرم الأمتزاز للعقار قيد البحث وبدرجات حرارية مختلفة ضمن الددى , 25 , 15 , 10

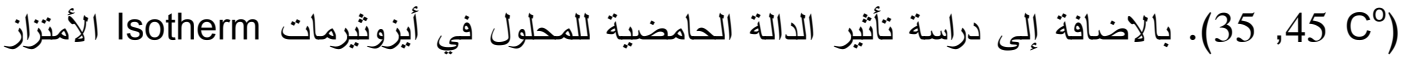

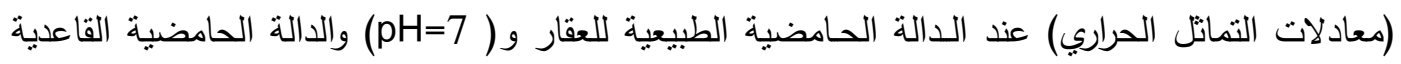

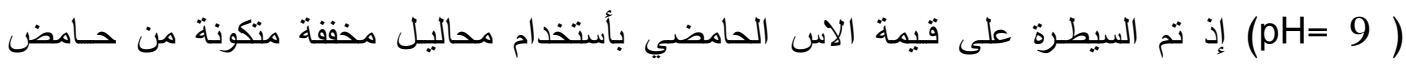

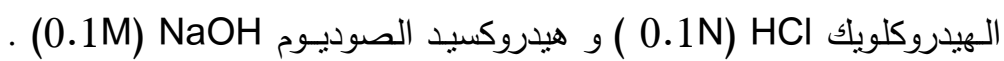

\section{النتائج والمناقشة}

لغرض منابعة عملية الامتزاز وتقدير كمية المادة الممتزة والمتنقية في المحلول ثم اللجوء إلى عمل

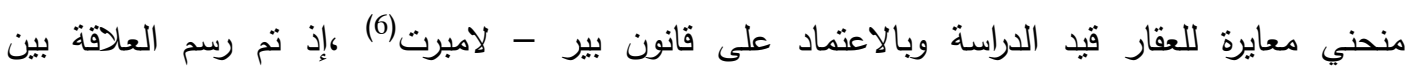
الامتصاصية والتركيز حيث اظهرت العلاقة خط مستقيم دلالة على خضوع هذا العقار لقانون بير - لامبرت

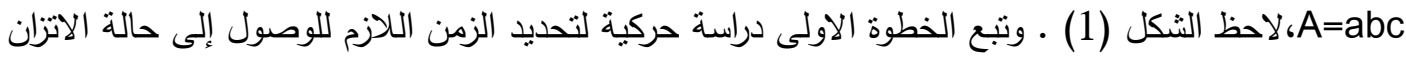

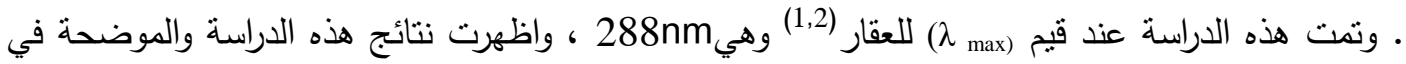

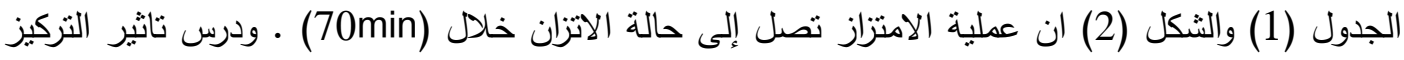

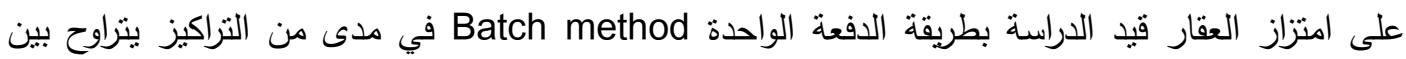

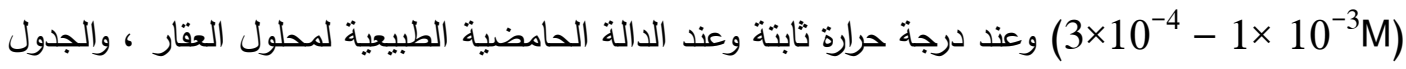
(2) يظهر نتائج هذه الدراسة . (2)

تثير النتائج الموضحة في الجدول (2) إلى ان لتركيز هذا العقار في المحلول ناثير مهما على عملية

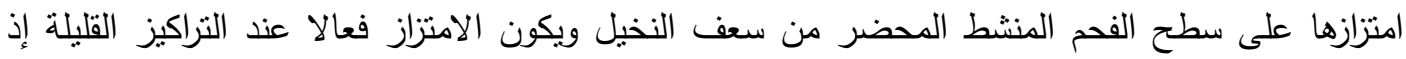
تختزل النسبة المئوية للامتزاز مع زيادة التركيز بسبب تسمم المسامات المنوفرة على سطح الفحم الدحضر (7). ولإجل تحديد الظروف المتلى لامتزاز العقار قيد الدراسة فقد تم اختبار امتزاز هذا العقار في اوساط

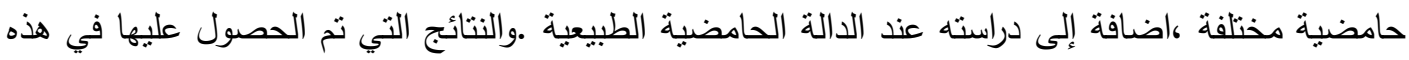

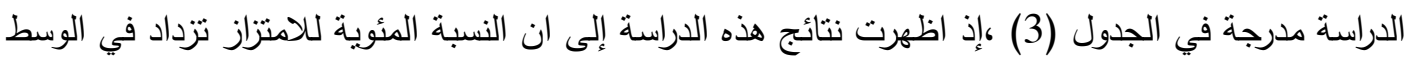


الحامضي ثم تتكرج بالنقصان في الوسط المتعادل ، فالوسط القاعدي • والسبب في هذا التتاقص يعود إلى الى

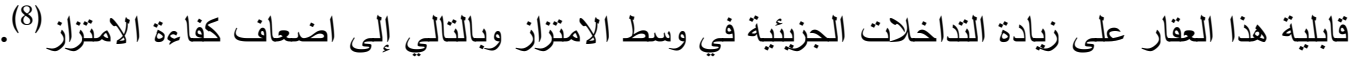

ودرس ناثير درجة الحرارة وفي مدى يتراوح بين(

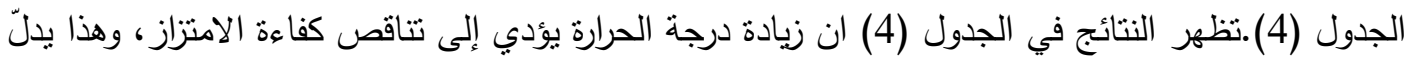

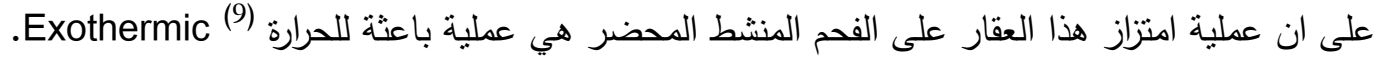
وتعد قيم الدوال الثزموديناميكية مهمة جدا في تفسير الكثير من التفاعلات من حيث اتجاه سيرها وعن طبيعة انظظام الجزيئات في الانظمة المختلفة الناتجة عن التداخلات الجزيئية بجميع انواعها ولاسيما عملية

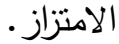

لقد نم حساب قيمة انثالبي الامتزاز باستخدام معادلة فانت هوف (Vant Hoff equation) والتي تمثل العلاقة بين ثابت التوازن ودرجة الحرارة .

$\mathrm{K}=\mathrm{K}_{\mathrm{O}} \mathrm{e}^{-\Delta \mathrm{H} / \mathrm{RT}}$

حيث تمثل هH حرارة الامتزاز (انثالبي الامتزاز ) و (K) ثابت التوازن على حين تمثل (Ko ثابتة .ومن خلال رسم العلاقة بين In K ومقلوب درجة الحرارة (1/T) الذي يعطي خطا مستقيا ميله يساوي ه وتمثل الثكل (3) الرسم الذي تم الحصول عليه وهو علاقة خطية وبمعامل

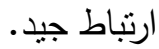

وبافتراض ان ثابت نوازن الامتزاز يمكن حسابه من النسبة بين تركيز المادة الممتزة وغير الممتزة في

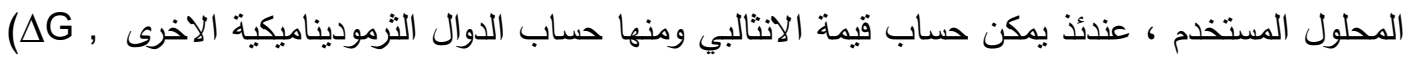
(11,10) من المعادلات الاتية

$\Delta \mathrm{G}=-\mathrm{RT} \ln \mathrm{K}$

$\Delta \mathrm{G}=\Delta \mathrm{H}-\mathrm{T} \Delta \mathrm{S}$

$\Delta S=\Delta H-\Delta G / T$ 
يمثل الجدول (5) قيم ثابت نوازن الامتزاز (K) عند درجات حرارية مختلفة وضمن المدى 15 , 10

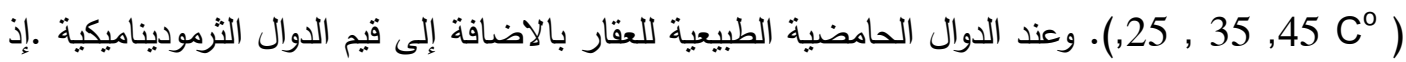

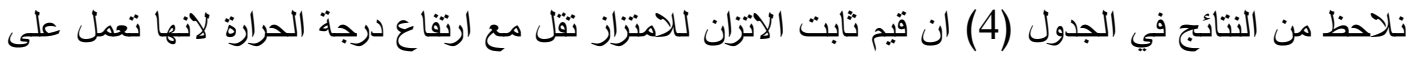

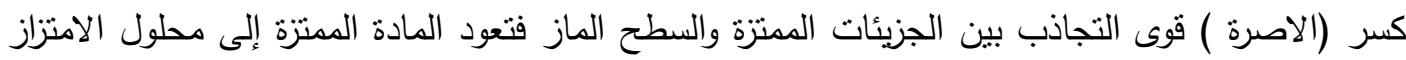
وبذللك تقل المادة الممتزة فتقل قيمة (K) تبعا لذلك ـ وان قيمة الانثالبي سالبة وهذا يدلّ على ان عملية

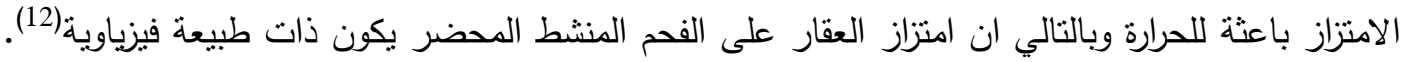

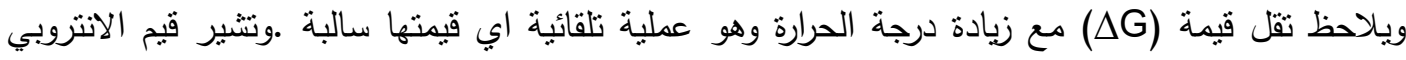

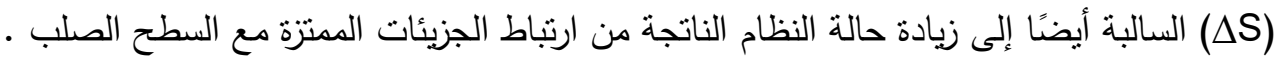

وتم استخدام معادلات الايزوثيرمات لنطويع البيانات العملية للامتزاز وتطبيقها عليها لحساب عدد من القيم والدوال الضرورية لوصف العلاقة بين المادة الممتزة والسطح ودراسة كفاءة الامتزاز ، وتعطي معادلات الايزوثيرمات عند تطبيقها وفي حالاتها المثالية علاقات خطية ـ وتم في هذا البحث استعمال ايزوثيرمين من

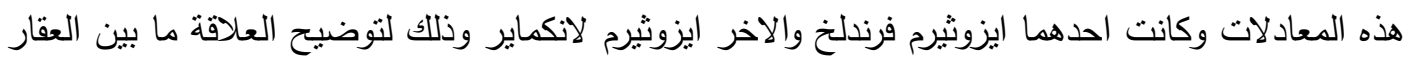

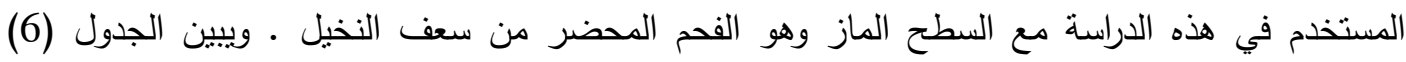

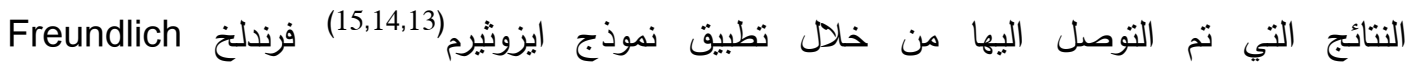
ولانكماير Langmuir ، وذللك باستخدام المعادلات الاتية :

$\log q_{e q}=\log K+1 / n \operatorname{loq} C_{e q}$

$\mathrm{C}_{\text {eq }} / \mathrm{q}_{\mathrm{eq}}=1 / \mathrm{bQ}+\mathrm{C}_{\text {eq }} / \mathrm{Q}$

إذ أعطى اختبار تطبيق كلا النموذجين للعقار قيد الدراسة علاقات خطية وبمعامل ارتباط جيد وكما في

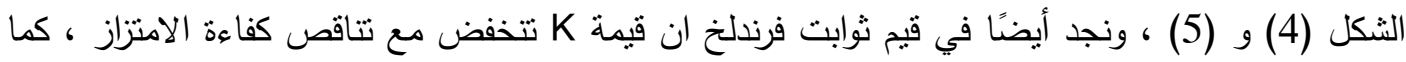

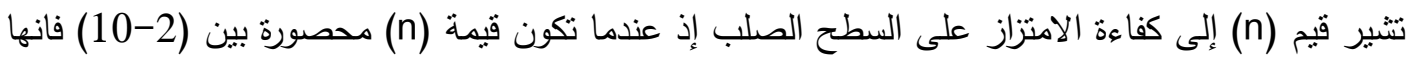

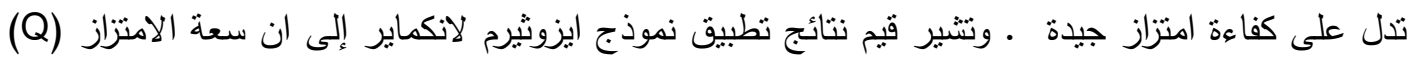

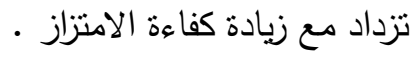




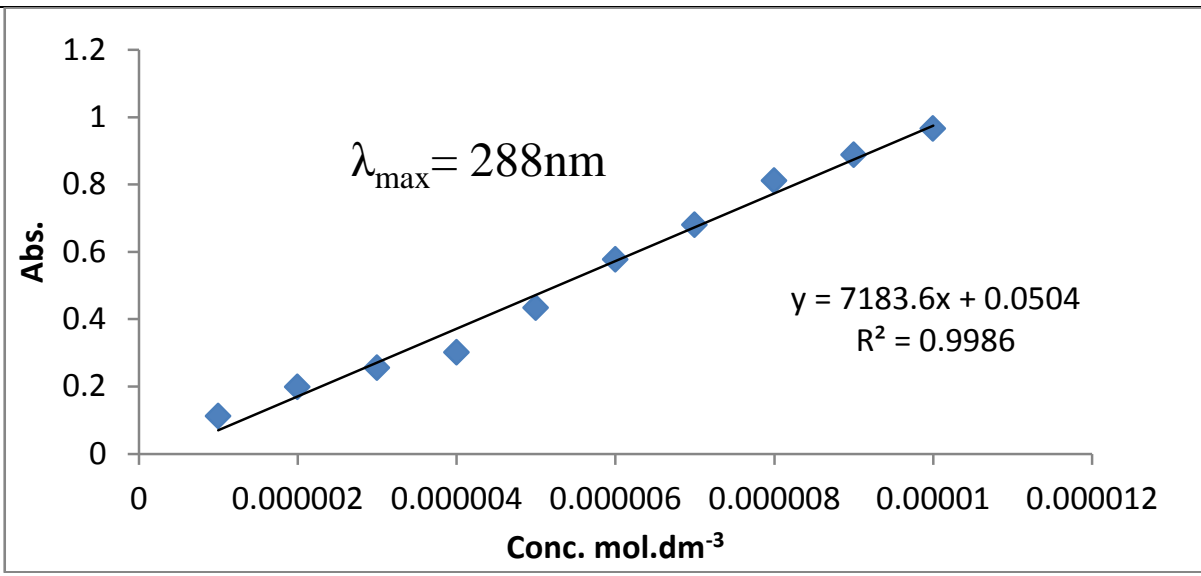

شكل (1):منحني المعايرة للعقار قيد الدراسة

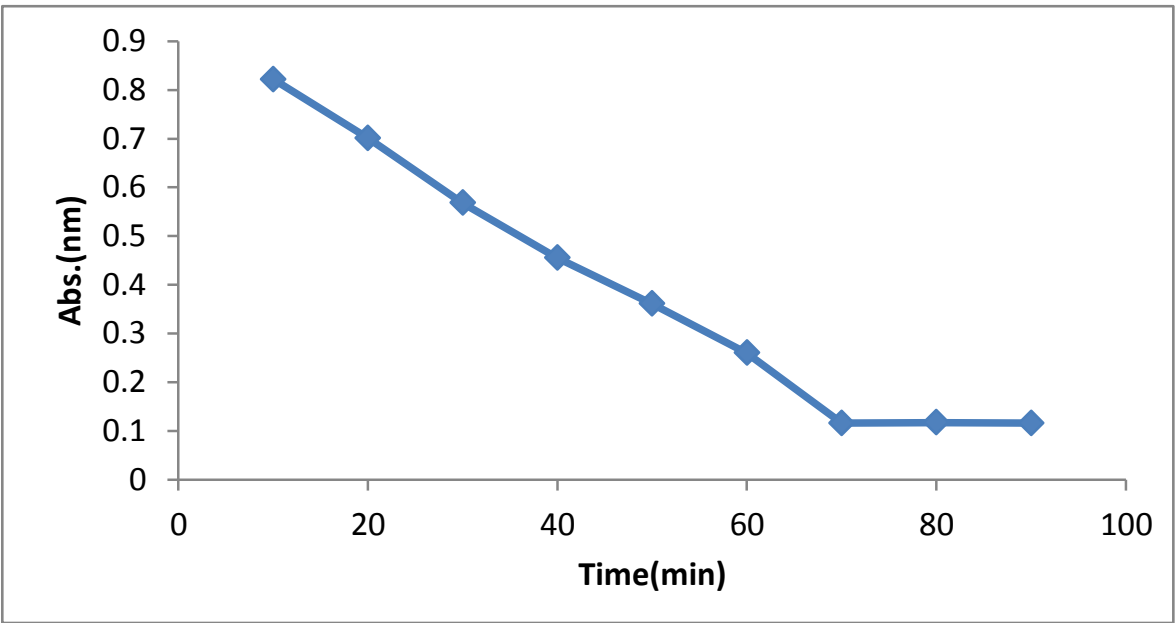

الثكل ( 2):تغير الامتصاص للعقار Mefenamic acid مع الزمن 
جدول (1) :تغير الامتصاص للعقار مع الزمن بتركيز (10 M 3 (10 ) مولاري

و وزن المادة المازة (0.2g)

\begin{tabular}{|c|c|c|c|c|c|c|c|c|c|}
\hline Time $(\mathrm{min})$ & 10 & 20 & 30 & 40 & 50 & 60 & 70 & 80 & 90 \\
\hline Abs. $(\mathrm{nm})$ & 0.822 & 0.701 & 0.568 & 0.455 & 0.361 & 0.260 & 0.116 & 0.117 & 0.116 \\
\hline
\end{tabular}

الجدول (2):ناثير التركيز على النسبة المئوية للامتزاز عند 283.15K

\begin{tabular}{|c|c|c|c|c|}
\hline Conc. (M) & $3 \times 10^{-4}$ & $5 \times 10^{-4}$ & $7 \times 10^{-4}$ & $1 \times 10^{-4}$ \\
\hline Abs. \% & 94.1 & 91.5 & 84.4 & 61.3 \\
\hline
\end{tabular}

Mefenamic acid الجدول (3):تاثير الدالة الحامضية على امتزاز

\begin{tabular}{|c|c|c|}
\hline Natural $^{*} \mathrm{pH}$ & $\mathrm{pH}=7$ & $\mathrm{pH}=9$ \\
\hline 97.5 & 94 & 81.4 \\
\hline
\end{tabular}

2.الدالة الحامضية الطبيعية للعقار 2.89

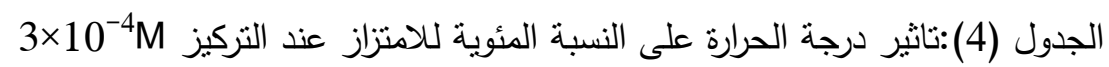

\begin{tabular}{|c|c|c|c|c|}
\hline \multicolumn{5}{|c|}{ \%of adsorption at temperature (K) } \\
\hline 283.15 & 288.15 & 298.15 & 308.15 & 318.15 \\
\hline 94.1 & 93.5 & 92 & 90.8 & 88.5 \\
\hline
\end{tabular}


العـــــــد الرابع والعشرون

مجلــــة كليــــة التربيــــة

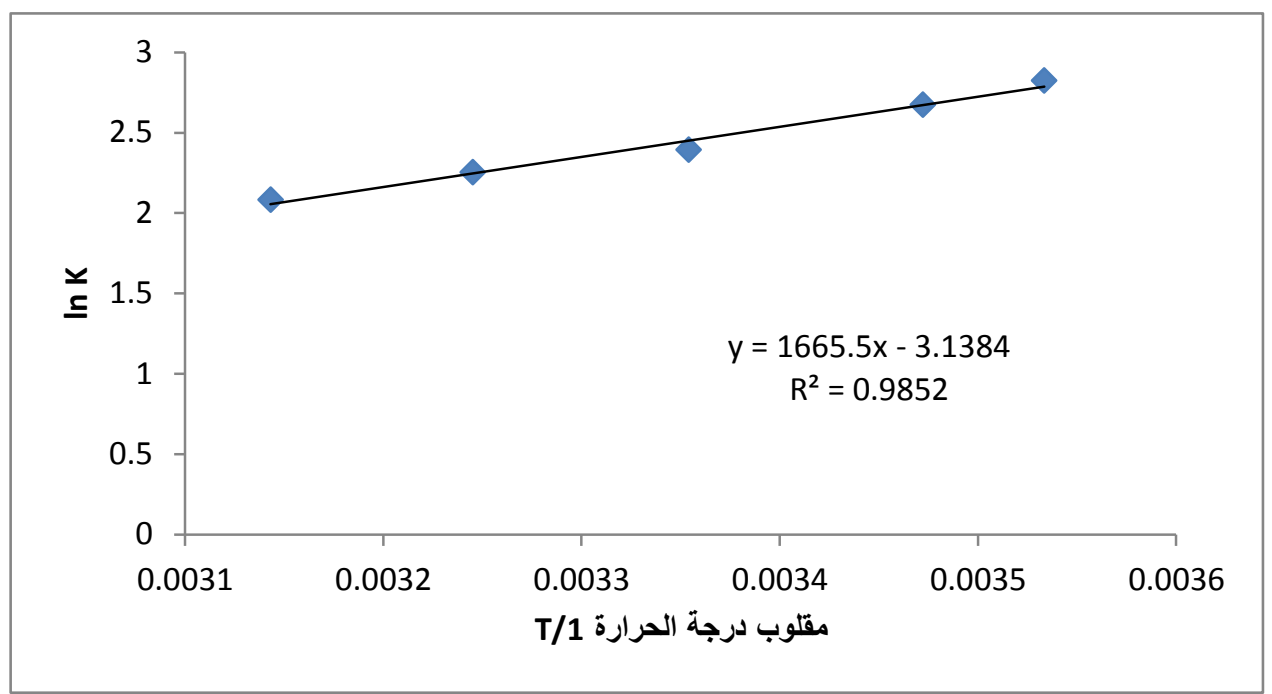

الثكل (3):العلاقة بين In K ومقلوب درجة الحرارة (1/T) لحساب قيمة انثالبي الامتزاز على الفحم المحضر عند الاتزان للعقار

الجدول (5): قيم الدوال الثرموداينميكية عند الاتزان للامتزاز على الفحم المضر

\begin{tabular}{|c|c|c|c|}
\hline $\begin{array}{c}\mathrm{T} \\
\left(\mathrm{K}^{\mathrm{O}}\right)\end{array}$ & $\begin{array}{c}\Delta \mathrm{G} \\
\left(\mathrm{J} . \mathrm{mole}^{-1}\right)\end{array}$ & $\begin{array}{c}\Delta \mathrm{H} \\
\left(\mathrm{J} . \mathrm{mole}^{-1}\right)\end{array}$ & $\begin{array}{c}\Delta \mathrm{S} \\
\left(\mathrm{J} . \mathrm{mole}^{-1} \cdot \mathrm{K}^{-1}\right)\end{array}$ \\
\hline 283.15 & -6472.7 & \multirow{5}{*}{-13846.99} & -26.1 \\
\hline 288.15 & -6303.5 & & -26.19 \\
\hline 298.15 & -6050.2 & & -26.14 \\
\hline 308.15 & -5892.2 & & -25.80 \\
\hline 318.15 & -5496.1 & & -26.20 \\
\hline
\end{tabular}


العــــــد الرابع والعشرون

مجلــــة كليــــة التربيـــة

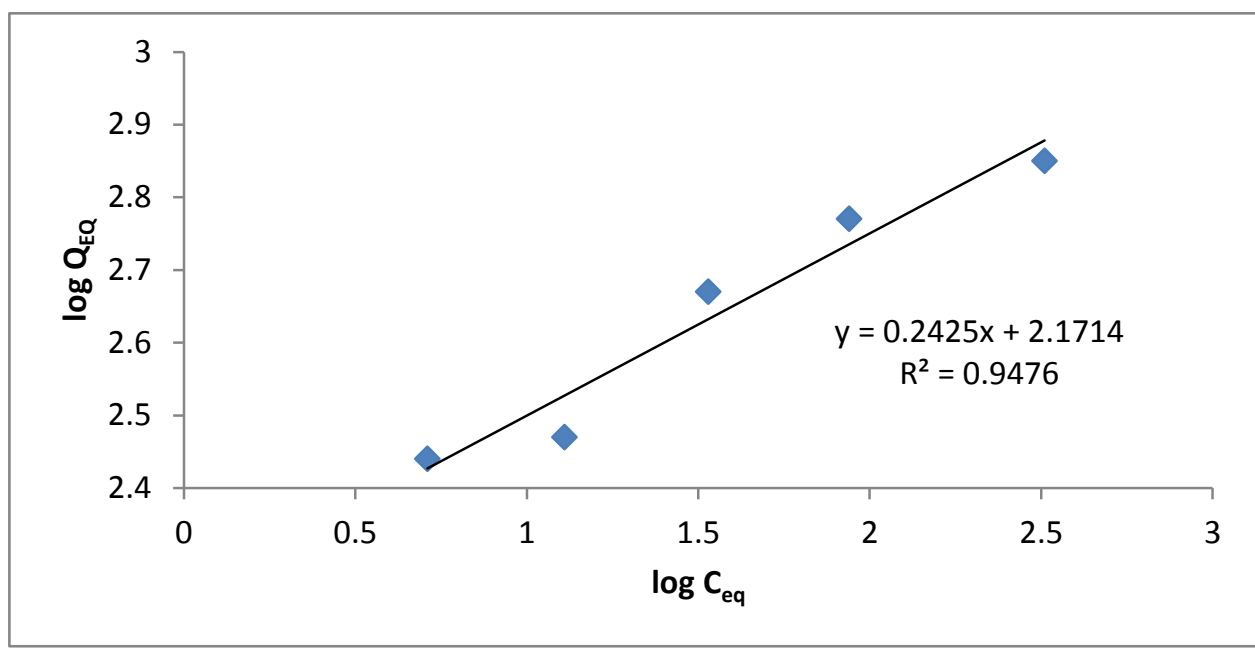

الثكل (4):ايزوثيرم فرندلخ لامتزاز Mefenamic acid على الفحم المحضر

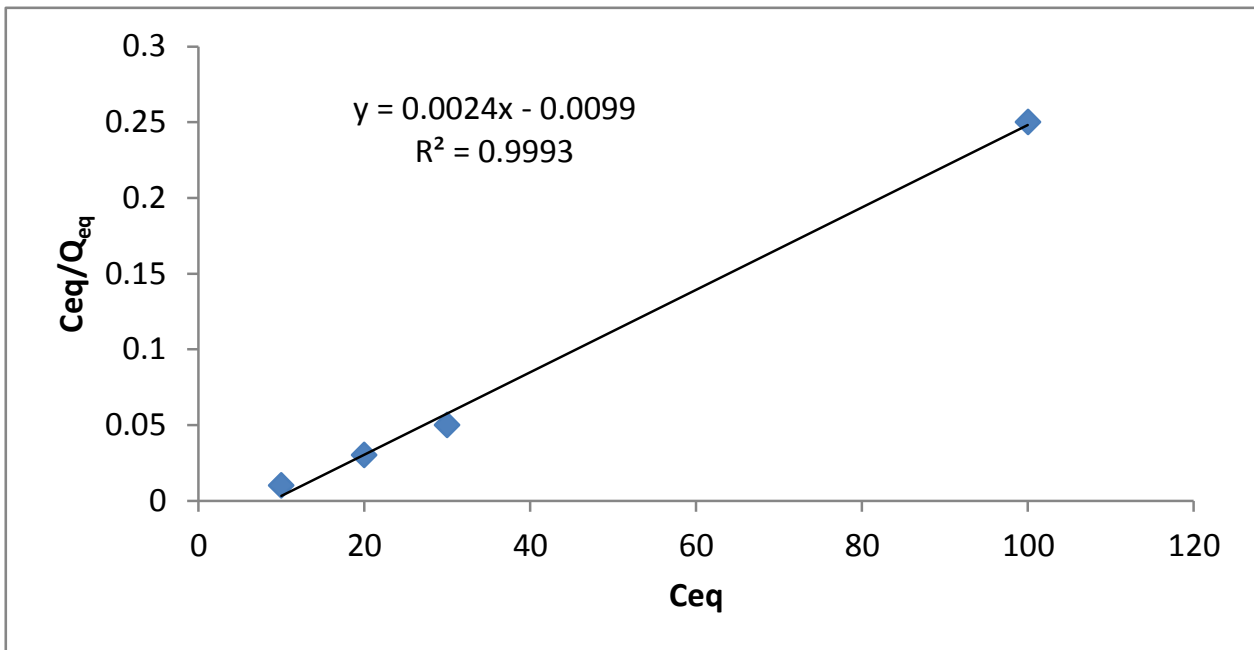

الثكل (5):ايزوثيرم لانكماير لامتزاز Mefenamic acid على الفحم المحضر

الجدول (6):قيم ثوابت فرندلخ ولانكماير ومعاملات الارتباط للامتزاز

\begin{tabular}{|c|c|c|c|c|c|}
\hline $\mathrm{N}$ & $\mathrm{K}$ & $\mathrm{R}$ & $\mathrm{Q}(\mathrm{mg} / \mathrm{g})$ & $\mathrm{b}(\mathrm{L} / \mathrm{mg})$ & $\mathrm{R}$ \\
\hline 4.12 & 148.4 & 0.9734 & 416.7 & 0.24 & 0.9996 \\
& & & & & \\
\hline
\end{tabular}




\section{reference}

1- H.G.Rolfe,"British pharmaceutical Codex ",1973.

2- "British pharmscopoeia ", International edition London : Mhso,(2001).

3- H. K. Abdul Hussein, "efficacy of attapulgite clay as adsorbent for metronidazole drug overdose in vitro'., national Journal of chemistry, volume 20, 2005,P.529-539.

4- R. A. Al-Bayati ,"Adsorption - Desorption of Trimethoprim Antibiotic Drug from Aqueous Solution by Two Different Natural Occurring Adsorbents ",2011,vol.3,No.3,P.9 .

5- E.D.Ahmed,"study adsorption durg paracetol on kaolin surface", No.106,Uinvercity of Tikrite ,(2013).

$$
\text { צ- ع.ع .الحيدري ،"التحليل الكيميائي الالي "، جامعة بغداد ، (1992). }
$$

7- M.M.Aslam and A. Matin ,"Removal of copper from industrial effluent by adsorption with economil viable material " ,Institute of Environmental Sciences and Engineering (IESE), National University of Sciences and Technology (NUST) . Tamiz-Ud-Din Road Raqalpindi Cantt . Pakistan , (2003) ,P. 1-8 .

8- H.M. Al-Obady B.G. Al-Shimmery, "The study of using Banana pseudo stem fibers to remove some dyes of polluted water", University of AlKuaf,No.1,(2012),P.12-22

9- R.Morrison and R.Boyed, "Organic Chemistry ", Allyn and Bacon,Inc. ,2 $2^{\text {nd }}$ ed. ,Boston.,(1968), P.772-777.

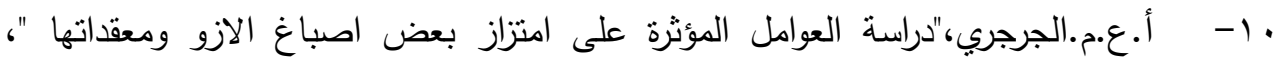

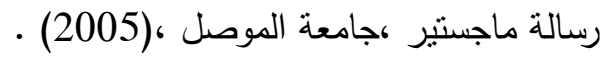

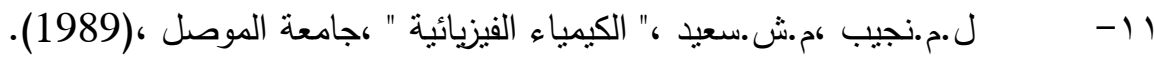

12- $\quad$ Adamson A.," Physical Chemistry of Surfaces", 4th Ed., Wiley-Inter science Publish ,(1984), P. 369-398. 
13-I. Langmuir, " The adsorption of gases on plane surfaces of glass mica and platinum ", J.Am. Chem Soc., 40, (1916),P. 1361-1403.

14. K.Laidlor and J.Meiser, "Physical Chem." ,Benjamin cumnings publishing company, Calisornia , (1982), P.775 .

15.W..Mook , M..Aroua and M.Szlachta ,"Palm Shell-based Activated

Carbon for removing reactive black 5 dye :Equilibrium and Kinetics Studies " ,Bio Resources 11(1), (2016) , P.1432-1447. 


\title{
Activated charcoal Preparation of Palm Fronds as a new
} adsorbent to remove case of poisoning drugs Mefenamic acid

\author{
Liqaa Hussin Al-waan Al-sammrae \\ College of Education-Sammara University
}

Drug overdose may treated via adsorbents especially via activated carbon hang to prohibit further absorption, Hence in this work a study was executed to rating the capability of Palm fronds as adsorbent for Mefenamic acid (ponstan).

Ultraviolet-Visible spectrophotometry technique was utilized to follow the volume or quantity of the adsorbed drug. The adsorption experiments were repeated at $\left(10,15,25,35,45 \mathrm{C}^{\mathrm{o}}\right)$ to measure the thermodynamical parameters $\left(\Delta \mathrm{H}^{\mathrm{o}}, \Delta \mathrm{G}^{\mathrm{o}}, \Delta \mathrm{S}^{\mathrm{o}}\right)$.

Mefenamic acid drug can be adsorbed via rebuilding carbon surface at depressed concentration of the drug and the quantity of the adsorbed drug increased with increasing the primary concentration of the drug. The adsorption quantities were lower with increasing temperature signal an exothermic adsorption operation which as well fortified via the account of thermodynamic parameters . Acidity has a small impact on adsorption . 